in vivo $32: 33-40(2018)$

doi:10.21873/invivo.11201

\title{
Effect of Benifuuki Tea on Cytochrome P450-mediated Metabolic Activity in Rats
}

\author{
TAKAHITO HIRAI ${ }^{1}$, YUKI NISHIMURA ${ }^{1}$, NORIMITSU KURATA ${ }^{2}$, HOKUTO NAMBA $^{3}$, MARIKO IWASE ${ }^{1}$, \\ YURIKA GOMI ${ }^{1}$, HIROMICHI TSUCHIYA ${ }^{4}$, TOMOYUKI YAMAKAWA ${ }^{1}$ and YUJI KIUCHI ${ }^{1}$ \\ ${ }^{1}$ Department of Pharmacology, Showa University, School of Medicine, Tokyo, Japan; \\ ${ }^{2}$ Faculty of Arts and Sciences at Fujiyoshida, Showa University, Fujiyoshida, Japan; \\ ${ }^{3}$ Showa University Clinical Research Institute for Clinical Pharmacology and Therapeutics, Tokyo, Japan; \\ ${ }^{4}$ Department of Medical Education, Showa University, School of Medicine, Tokyo, Japan
}

\begin{abstract}
Background/Aim: Benifuuki tea has recently been used as an alternative therapy for pollinosis, and it may be consumed with pharmaceutical drugs. This study aimed to examine cytochrome P450 (CYP)-mediated food-drug interactions with Benifuuki tea in rats. Materials and Methods: The inhibitory effects of Benifuuki tea and (-)-epigallocatechin-3-O-(3-O-methyl) gallate (EGCG3"Me) on $C Y P$ activities were evaluated in vitro. Midazolam pharmacokinetics was investigated after two treatments with Benifuuki tea. In an ex vivo study, CYP activities were determined after 1-week-treatment with the tea. Results: Benifuuki tea and EGCG3"Me inhibited CYP2D and CYP3A activities in a concentration-dependent manner in vitro. However, MDZ metabolism did not change by Benifuuki treatment in vivo and ex vivo. In contrast, CYP $2 D$ activity was decreased ex vivo. Conclusion: Normal intake of Benifuuki tea is not likely to cause food-drug interactions by CYP3A inhibition or induction. In contrast, Benifuuki tea consumption may lead to food-drug interactions through the inhibition of CYP2D.
\end{abstract}

Pollinosis is one of the most common allergic diseases in Japan, with an estimated morbidity of more than $20 \%$ (1-3). Patients with pollinosis experience symptoms such as sneezing and nasal discharge, which require long-term symptomatic treatment with antihistamines, $\alpha_{1}$ agonists, and steroids. However, the side-effects of these pharmaceutical

This article is freely accessible online.

Correspondence to: Yuki Nishimura, Department of Pharmacology, Showa University, School of Medicine, 1-5-8 Hatanodai, Shinagawa-ku, Tokyo 142-8555, Japan. Tel: +3 37848125, Fax: +3 37848176, e-mail: yukin@med.showa-u.ac.jp

Key Words: Cytochrome P450, Benifuuki tea, pharmacokinetics, food-drug interactions, CYP2D, CYP3A. products may reduce patients' quality of life. For this reason, patients often use health foods together with, or instead of, pharmaceutical drugs to treat pollinosis.

Benifuuki tea, a green tea cultivar (Camellia sinensis L.) has recently attracted attention as an alternative therapy for pollinosis. The major ingredients in green tea are known to be catechins. One of the main catechins in Yabukita tea, which is commonly consumed in Japan, is (-)-epigallocatechin gallate (EGCG). In addition to EGCG, Benifuuki tea contains another major catechin, namely (-)-epigallocatechin 3-(3"-O-methyl) gallate (EGCG3"Me) (4). EGCG3"Me has been shown to inhibit mast cell activation through the suppression of tyrosine phosphorylation of cellular proteins (5). Furthermore, it has been reported that EGCG3"Me inhibits histamine release from murine bone marrow mast cells more potently compared with EGCG (6). In fact, compared with Yabukita tea, Benifuuki tea has been shown to significantly relieve the symptoms of pollinosis in a double-blinded clinical trial (7). Therefore, in recent years, Benifuuki tea containing EGCG3"Me has become increasingly popular among patients with pollinosis.

Individuals with pollinosis may easily obtain Benifuuki tea at drug stores, grocery stores, or on the internet, and consume this tea over long periods; therefore, it is likely that its consumption is combined with regularly used medicines. Moreover, drug interactions between medicines and beverages such as herbal tea or fruit juice have been previously reported; for example, it has been shown that grapefruit juice elevates the blood levels of medicines such as nifedipine and simvastatin through the inhibition of cytochrome P450 (CYP) $3 \mathrm{~A}$ activity in the small intestine (8). Conversely, we showed that the ingestion of tenryocha tea or rooibos tea for 2 weeks reduced the area under the blood concentration-time curve (AUC) and the maximum serum concentration $\left(\mathrm{C}_{\max }\right)$ of midazolam (MDZ) by more than $60 \%$ thorough intestinal CYP3A induction in rats (9). Although most beverages are likely consumed together with other medicines, there are few reports on possible food-drug interactions, especially in vivo. 
Previous studies have described the effect of green tea on CYP3A activity: green tea extract (GTE) has been shown to inhibit CYP3A activity in the human liver and intestinal microsomes $(10,11)$. Furthermore, an in vivo study showed that 1 week of treatment with GTE in rats reduced intestinal CYP3A activity but increased hepatic CYP3A protein levels (11). GTE therefore shows both inhibitory and inducible effects on CYP3A activity, and EGCG has been suggested to be responsible for some of these effects (10-12).

As the degree of absorption of EGCG from the gastrointestinal tract is low, drug interactions mediated by GTE via CYP3A modulation are thought to occur mainly in the small intestine. The plasma EGCG level is likely low after drinking a normal quantity of green tea; therefore, it would be difficult to achieve to a level that would influence hepatic CYP3A activity. Conversely, the intake of EGCG 3"Me has been shown to result in higher AUC and bioavailability compared with orally administered EGCG in rats (13). Furthermore, a study of healthy human volunteers showed that the AUC of EGCG3"Me was higher than that of EGCG after Benifuuki tea ingestion (14). Therefore, if EGCG3"Me as well as EGCG influence CYP3A, Benifuuki tea, which contains both, is likely to have more potent inhibitory or inducible effects on CYP3A than Yabukita tea. However, there have been no reports on drug interactions mediated by Benifuuki tea via CYP3A modulation.

CYP isozymes other than CYP3A also play an important role in drug metabolism. It has been shown that GTE and EGCG inhibit CYP1A, CYP2C9, CYP2C19, and CYP2D6 activities in vitro (10-12). On the other hand, GTE has been shown to increase mRNA and protein levels of CYP1A and CYP2C in HepG2 cells (15). These CYP isoforms are expressed in the small intestine at very low levels, and drug interactions mediated by these enzymes mainly occur in the liver. Therefore, these in vitro drug interactions are less likely to occur in vivo when consuming green tea containing EGCG as its main ingredient, because of its low absorption from the intestinal tract. However, the effect of Benifuuki tea, which is rich in EGCG 3"Me, on these CYPs is unknown.

In this context, the current study was conducted on rats to determine whether the intake of a normal quantity of Benifuuki tea influences the metabolism of a co-administered medicine through the inhibition or induction of the main drug-metabolizing enzyme, CYP3A. Furthermore, we examined the effect of multiple treatments with Benifuuki tea on CYP1A, CYP2C, and CYP2D, which also play important roles in drug metabolism.

\section{Materials and Methods}

Chemicals and Benifuuki tea. MDZ maleate was kindly donated by Nippon Roche Co. Ltd. (Tokyo, Japan); 4-hydroxymidazolam and 7-hydroxywarfarin were purchased from Daiichi Pure Chemicals Co., Ltd. (Tokyo, Japan). Bufuralol and 1'-hydroxybufuralol were purchased from Gentest Corp. (Woburn, MA, USA), S-warfarin was from purchased Toronto Research Chemicals Inc. (Toronto, ON, Canada), resorufin and ethoxyresorufin were purchased from SigmaAldrich Co. (St. Louis, MO, USA), and nitrazepam and diazepam were purchased from Wako Pure Chemical Industries, Ltd. (Osaka, Japan). EGCG was obtained from Mitsui Norin Co. Ltd. (Shizuoka, Japan), and EGCG3"Me was purchased from Nagara Science Co. Ltd. (Gifu, Japan). All other chemicals were of analytical grade quality.

Polyethylene terephthalate (PET)-bottled Benifuuki tea containing $17.5 \mathrm{mg} / 500 \mathrm{~mL}$ EGCG3"Me was a kind gift from Akahori Shoten (Shizuoka, Japan).

Treatment of animals and sampling. Male Sprague-Dawley rats (Japan Laboratory Animals, Inc., Tokyo, Japan), weighing 220-250 g, were used in this study. The rats were housed in a temperaturecontrolled room with a 12-h light and dark cycle and fed standard laboratory chow. The rats were fasted overnight before the day of sampling. The procedures for this study were reviewed and approved by the Showa University Ethics Committee for Animal Care and Use, and conducted in accordance with guidelines established by the National Institute of Health guide for the care and use of Laboratory animals (8th edition).

In the two-treatment study, $10 \mathrm{ml} / \mathrm{kg}$ Benifuuki tea or water (control) was orally administered to rats $(\mathrm{n}=5)$ at 0.5 and $2 \mathrm{~h}$ before oral administration of MDZ $(20 \mathrm{mg} / \mathrm{kg})$. Blood samples $(200 \mu \mathrm{l})$ were collected from the jugular vein before and 15, 30, 45, 60, 90, 120,180 , and $240 \mathrm{~min}$ after MDZ administration. The samples were centrifuged at $7,500 \times g$ for $10 \mathrm{~min}$ at $4^{\circ} \mathrm{C}$, and each serum sample was stored at $-80^{\circ} \mathrm{C}$ until analysis.

In the 1 -week-treatment study, rats $(n=6)$ were administered 10 $\mathrm{ml} / \mathrm{kg}$ Benifuuki tea or water (control) orally once a day for 1 week. One day after the last Benifuuki tea treatment, the rats were sacrificed, and the liver and small intestine were excised. Liver microsomes were prepared based on a conventional fractional centrifugation method, and were suspended in $100 \mathrm{mM}$ potassium phosphate buffer ( $\mathrm{pH}$ 7.4) (16). Microsomal fractions were frozen in liquid nitrogen and stored at $-80^{\circ} \mathrm{C}$ until use. Protein concentrations were measured by the method of Lowry et al. (17).

Determination of serum midazolam concentration. After the two treatments with Benifuuki tea, MDZ serum concentrations were measured by an HPLC method previously reported by our laboratory $(18,19)$. Briefly, $100 \mu \mathrm{l}$ of each serum sample was alkalized and extracted with dichloromethane: pentane (1:1) for 1 min in the presence of diazepam as an internal standard. After the evaporation of the organic phase, the dried residue was dissolved in the mobile phase and injected onto an HPLC apparatus equipped with a CAPCELLPAK C18 MGII column $(2.0 \mathrm{~mm} \times 250 \mathrm{~mm}$, Shiseido, Tokyo, Japan). The mobile phase consisted of $10 \mathrm{mM}$ potassium phosphate buffer ( $\mathrm{pH} 5.0$ ): acetonitrile (50:50), and the flow rate was $0.2 \mathrm{ml} / \mathrm{min}$. Peaks were detected using a UV detector at $220 \mathrm{~nm}$.

Pharmacokinetic analysis. The pharmacokinetic parameters of MDZ were obtained by non-compartmental analysis using MOMENT $\left(\right.$ Excel $\left.^{\circledR}\right)$ based on the moment analytic method $(20,21)$. $\mathrm{AUC}_{0-\infty}$ was calculated according to the trapezoidal rule. The apparent elimination rate constant $(\mathrm{Ke})$ was determined from the gradient of the elimination phase, and the elimination half-life $\left(t_{1 / 2}\right)$ was calculated as $\ln 2 / \mathrm{Ke}$. $\mathrm{C}_{\max }$ was obtained from the actual data. 
Assay for CYP activities. Liver microsomes, prepared 1 day after the last treatment in the 1-week Benifuuki tea administration experiment, were used in the ex vivo study. Ethoxyresorufin O-deethylation (EROD), S-warfarin 7-hydroxylation (S-WF 7-OH), bufuralol 1'hydroxylation (BF 1'-OH), and MDZ 4-hydroxylation (MDZ 4-OH) activities were assayed as the oxidation markers for CYP1A, CYP2C, CYP2D, and CYP3A, respectively. EROD was assayed fluorometrically according to methods described previously (22). SWF 7-OH, BF 1'-OH, and MDZ 4-OH were assayed, and each metabolite was determined by HPLC as described previously (23-25).

An in vitro study was also carried out to investigate the inhibitory effects of Benifuuki tea, EGCG3"Me, or EGCG on CYP2D and CYP3A activities using pooled rat liver microsomes, which were prepared from 10 non-treated male Sprague-Dawley rats. The PETbottled Benifuuki tea was established as $100 \%$ tea and was used after dilution to $1-10 \%$ as the final concentration. EGCG3"Me and EGCG were used at a concentration of 6-600 $\mu \mathrm{M}$. Each control activity was also determined under the same conditions, but with the addition of water instead of the tea or catechins. Data are expressed as the percentage of the remaining activities in the control experiments. $\mathrm{IC}_{50}$ values were estimated through the nonlinear regression of the relative reaction velocities at a single substrate concentration in the presence of varying EGCG3"Me or EGCG concentrations.

In addition, preincubation experiments were performed to determine whether any produced metabolic intermediates inactivated CYP2D and CYP3A. Benifuuki tea (CYP2D: 0.5\%, CYP3A: $5 \%$ ) was preincubated with rat liver microsomes at $37^{\circ} \mathrm{C}$ for 5-15 min for BF 1' $-\mathrm{OH}$, and for 10-30 min for MDZ 4-OH, in the presence or absence of NADPH. After the preincubation step, each substrate was added, and the activities were assayed as described above.

Measurement of mRNA expression. A total of 20-30 mg of liver and intestinal tissues, excised 1 day after the last treatment in the 1-week Benifuuki tea administration study, were used to determine the expression of CYP mRNAs. Total RNA was extracted from these samples using an RNeasy Mini kit (Qiagen Inc., CA, USA) according to the protocol provided by the manufacturer. The concentration of RNA was determined by spectrophotometry at $260 \mathrm{~nm}$. RNA samples (900 ng) were reverse-transcribed using the High Capacity RNA-to-cDNA master Mix (Applied Biosystems Inc., CA, USA) to obtain cDNA, according to the manufacturer's recommendations. cDNA $(2 \mu \mathrm{l})$ was amplified using CYP2D2-, CYP3A2-, or CYP3A9-specific TaqMan primer and probe sets (TaqMan Gene Expression Assays, Applied Biosystems Inc., CA, USA) in a total volume of $20 \mu \mathrm{l}$. Real-time PCR analysis was performed to quantify the relative $C Y P 2 D 2, C Y P 3 A 2$, or $C Y P 3 A 9$ mRNA expression using Applied Biosystems StepOne ${ }^{\mathrm{TM}}$. GAPDH levels were monitored as an endogenous control. PCR reactions included an initial denaturizing cycle at $95^{\circ} \mathrm{C}$ for $10 \mathrm{~min}$, followed by 40 amplification cycles of $95^{\circ} \mathrm{C}$ for $15 \mathrm{~s}$ and $60^{\circ} \mathrm{C}$ for $1 \mathrm{~min}$. Relative-fold mRNA expression was determined for each sample using the $2^{-\Delta \Delta C T}$ method.

Statistical analyses. In vivo and ex vivo data are presented as the mean \pm standard deviation (S.D.). In vitro data are expressed as the mean \pm standard error (S.E.) of duplicate examinations. The significance was determined by applying Mann-Whitney $U$-test, or one-way analysis of variance with Dunnett post hoc test, as appropriate. A $p$-value with $<0.05$ was considered to be significant.

\section{Results}

CYP3A activity in vitro. Benifuuki tea (1-10\%) inhibited CYP3A-mediated MDZ 4-OH activity in pooled rat liver and small intestinal microsomes in a concentration-dependent manner (Figure 1A and B). Furthermore, EGCG3"Me, which is a major catechin present in Benifuuki tea, inhibited hepatic and small intestinal CYP3A activities, with $\mathrm{IC}_{50}$ values of $69 \mu \mathrm{M}$ and $72 \mu \mathrm{M}$, respectively (Figure $1 \mathrm{D}$ and E). The potency of the inhibition was similar to that of EGCG on liver CYP3A activity $\left(\mathrm{IC}_{50}=74 \mu \mathrm{M}\right)$ (Figure $\left.1 \mathrm{~F}\right)$.

To identify the possible contribution of active intermediates to the inhibitory effect of Benifuuki tea on CYP3A activity, another inhibition study that included a preincubation step was conducted. The results indicated that the inhibition potency of Benifuuki tea on CYP3A activity did not depend on NADPH or the preincubation time (Figure 1C).

MDZ pharmacokinetics. Benifuuki tea was administered twice at $0.5 \mathrm{~h}$ and $2 \mathrm{~h}$ before MDZ administration; then, MDZ pharmacokinetics were compared with those of the control group treated with water. The serum MDZ concentration-time profiles and the pharmacokinetic parameters are shown in Figure 2 and Table I. No significant differences in serum MDZ level and its $\mathrm{AUC}_{0-\infty}, \mathrm{C}_{\text {max }}$, and $t_{1 / 2}$ values were found between the two groups.

CYP activities and mRNA expression ex vivo. After 1 week of treatment with Benifuuki tea, liver and intestinal tissues were excised, and CYP activities were analyzed. Benifuuki tea treatment did not increase hepatic CYP activities through the induction of these enzymes (Figure 3A, B and C, 3D). In contrast, CYP2D-mediated BFL 1'-OH was significantly inhibited by $20 \%$ in the Benifuuki tea treatment group $(p<0.05)$ (Figure 3C). On the other hand, the mRNA expression levels of liver CYP2D2, liver CYP3A2, and intestinal CYP3A9 after Benifuuki tea treatment were similar to those of the controls (Figure 3E, F and G).

CYP2D activity in vitro. Benifuuki tea (1-10\%) inhibited hepatic BFL 1'-OH activity in a concentration-dependent manner (Figure 4A). Furthermore, EGCG 3"Me and EGCG both inhibited BFL 1'-OH activity, with $\mathrm{IC}_{50}$ values of $65 \mu \mathrm{M}$ for each (Figure $4 \mathrm{~B}$ and $\mathrm{C}$ ). Meanwhile, in the preincubation study, there was no remarkable change in the potency of inhibition of Benifuuki tea on BFL 1'-OH activity following the preincubation step (Figure 4D).

\section{Discussion}

In the current study, we examined the effect of Benifuuki tea, which is used to relieve the symptoms of pollinosis, on CYPmediated food-drug interactions. 


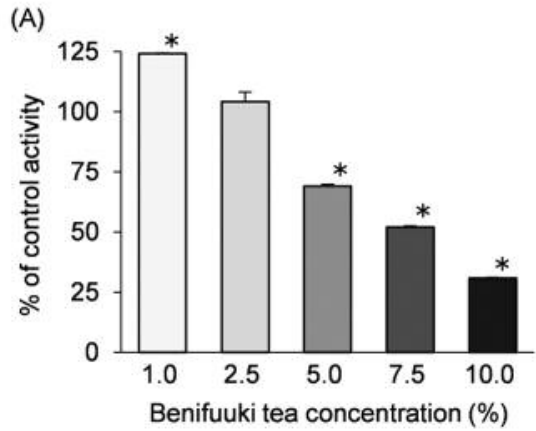

(D)

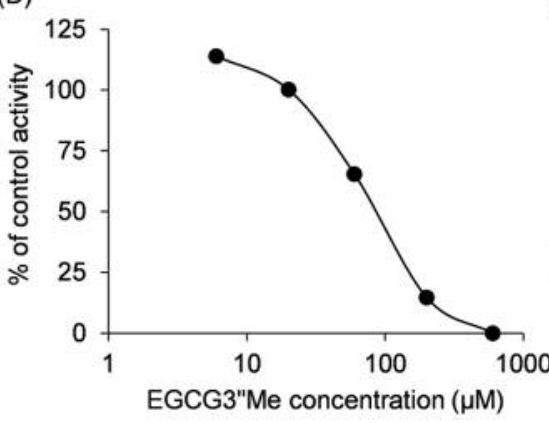

(B)

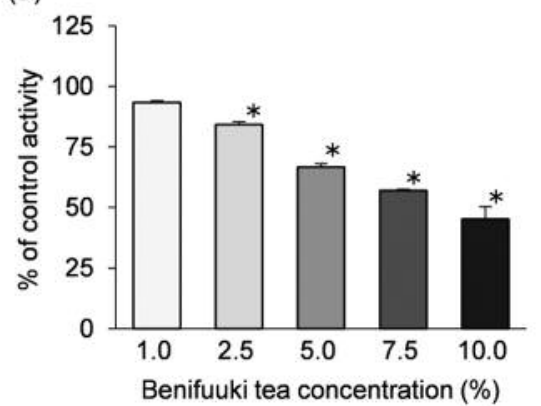

(E)

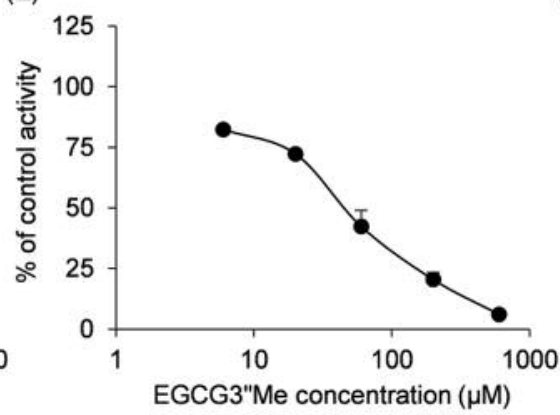

(C)

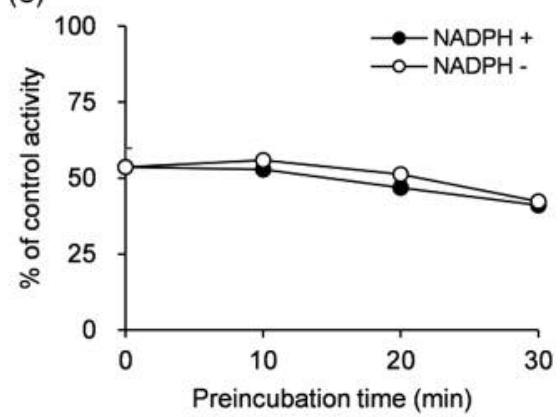

(F)

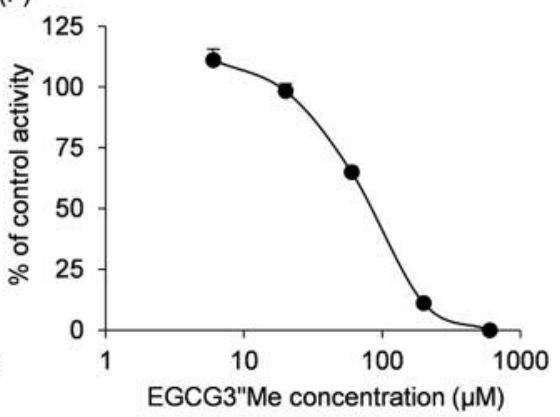

Figure 1. Inhibitory effect of Benifuuki tea, EGCG3"Me, and EGCG on MDZ 4-OH activity in vitro. A direct inhibition study with Benifuuki tea was performed in pooled rat liver (A) or intestinal (B) microsomes. The effect of EGCG3"Me in pooled rat liver (D) or intestinal (E) microsomes, and that of EGCG in pooled rat liver microsomes $(F)$, was also studied. Pre-incubation effect of Benifuuki tea (5\%) on MDZ 4-OH activity was studied using pooled rat liver microsomes in the presence (closed circles) or absence (opened circles) of NADPH (C). The mean $\pm S . E$. values of duplicate examinations are shown as a percentage of the control activity. ${ }^{*} p<0.05$ compared to control.

First, the inhibitory effects of Benifuuki tea and its main ingredient, EGCG3"Me, on CYP3A activity were investigated in vitro using $\mathrm{MDZ} 4-\mathrm{OH}$ as a marker of CYP3A-mediated metabolism. The results indicated that Benifuuki tea and EGCG3"Me inhibited MDZ-4OH activity in rat liver and intestinal microsomes in a concentrationdependent manner (Figure 1). The potency of the inhibition of hepatic CYP3A activity by EGCG3"Me $\left(\mathrm{IC}_{50}=69 \mu \mathrm{M}\right)$ was similar to that by EGCG $\left(\mathrm{IC}_{50}=74 \mu \mathrm{M}\right)$ (Figure $1 \mathrm{D}$ and F). It has been reported that EGCG is the most abundant of the catechins in green tea and the most effective inhibitor of CYPs in vitro (26). Our results showed that, like common green tea (Yabukita tea), Benifuuki tea inhibited CYP3A activity. In addition to EGCG, Benifuuki tea contains a high level of EGCG3"Me, an ingredient considered to have an inhibitory effect on this enzyme.

The Benifuuki tea we used in this study contained $35 \mu \mathrm{g} / \mathrm{ml}$ (74 $\mu \mathrm{M})$ EGCG3"Me and $17.5 \mu \mathrm{g} / \mathrm{ml}(38 \mu \mathrm{M})$ EGCG (data not shown). Accordingly, $10 \%$ Benifuuki tea, which inhibited hepatic CYP3A activity by more than $50 \%$ in vitro, contained 7.4 $\mu \mathrm{M}$ EGCG3"Me and 3.8 $\mu \mathrm{M}$ EGCG. These concentrations of EGCG3"Me and EGCG did not have any inhibitory effects on CYP3A activity (Figure 1D and F). Thus, it was concluded that, in addition to EGCG3"Me and EGCG, Benifuuki tea contains ingredients that inhibit CYP3A activity; all of these compounds were considered to be involved in the inhibitory effect.

We then investigated the effect of Benifuuki tea on MDZ pharmacokinetics in rats to determine whether the tea caused drug interactions through CYP3A inhibition in vivo. Two treatments with Benifuuki tea, at $0.5 \mathrm{~h}$ and $2 \mathrm{~h}$, before MDZ administration did not cause any remarkable change in the MDZ pharmacokinetic parameters (Figure 2, Table I). Nishikawa et al. reported that a single oral treatment with $400 \mathrm{mg} / \mathrm{kg}$ GTE, which contains a considerably higher amount of EGCG than green tea, did not change blood MDZ concentrations (11). Compared with EGCG, EGCG3"Me is thought to reach at a high enough level to the liver; therefore, it was hypothesized that Benifuuki tea inhibits hepatic CYP3A activity. However, no significant increase in serum MDZ concentration was observed in vivo. Therefore, it was concluded that the quantities of these CYP3A inhibitors present in the liver and small intestine after two treatments with Benifuuki tea were insufficient to inhibit CYP3A. The quantity of Benifuuki tea administered to rats in this study $(10 \mathrm{ml} / \mathrm{kg})$ was estimated to be equal to the 
Table I. Pharmacokinetic parameters of MDZ after two treatments with Benifuuki tea in rats.

\begin{tabular}{lccc}
\hline & $\begin{array}{c}\mathrm{AUC}_{0-\infty} \\
\left(\mathrm{ng} / \mathrm{mL}^{*} \mathrm{~h}\right)\end{array}$ & $\begin{array}{c}\mathrm{C}_{\max } \\
(\mathrm{ng} / \mathrm{mL})\end{array}$ & $\begin{array}{c}\mathrm{t}_{1 / 2} \\
(\mathrm{~min})\end{array}$ \\
\hline Control & $2,332 \pm 108$ & $2,223 \pm 199$ & $34 \pm 12$ \\
Benifuuki tea & $2,773 \pm 830$ & $2,365 \pm 644$ & $33 \pm 14$ \\
\hline
\end{tabular}

Each value represents the mean \pm S.D. for 5 rats.

level humans generally consume in one serving. Thus, the results suggest that the food-drug interactions mediated by CYP3A inhibition likely do not occur as a result of the intake of one normal serving of Benifuuki tea.

However, Benifuuki tea is often consumed over a long period to relieve the symptoms of pollinosis. It has been reported that St. John's Wort induces CYP3A in vivo, despite inhibiting the activity of this enzyme in vitro $(27,28)$. There is little information on food-drug interactions involving CYP induction. In order to ensure the effective use of medicines, it is important to investigate the decrease in efficacy caused by the reduction in their concentrations in the blood. We examined the inducible effects of 1 week of treatment with Benifuuki tea on hepatic CYP activities and the intestinal mRNA level of CYP3A9, which is predominantly expressed in the rat small intestine. The results indicated no significant increase in hepatic CYP1A, CYP2C, CYP2D, and CYP3A activities or hepatic CYP2D2, hepatic CYP3A2, and intestinal CYP3A9 mRNA levels following the tea treatment (Figure 3). These results suggest that Benifuuki tea does not elicit the induction of CYPs mainly involved in drug metabolism in the liver and small intestine. Nishikawa et al. reported that, in rats, 1 week of treatment with GTE increased hepatic CYP3A protein level by 1.5 times but reduced intestinal CYP3A activity (11). Nishikawa et al. used GTE containing a high amount of EGCG, whose levels must have been sufficient to modulate hepatic CYP3A. Conversely, as shown in the present in vivo study, normal intake of Benifuuki tea for 1 week did not have an inducible effect on CYPs.

In contrast, a $20 \%$ decrease in hepatic CYP2D-mediated BFL 1'-OH activity unexpectedly occurred after Benifuuki tea treatment for 1 week (Figure 3). Therefore, we investigated the possibility of down-regulation of hepatic CYP2D by measuring its mRNA expression. The results indicated that the CYP2D2 mRNA level was the same as that of the control group. In other words, CYP2D activity was inhibited by 1 week of treatment with Benifuuki tea (even though this was measured 1 day after the last tea treatment) without a reduction in CYP2D2 mRNA expression. It was previously reported that GTE and EGCG have no or a minor inhibitory effect on CYP2D activity both in vitro and in vivo

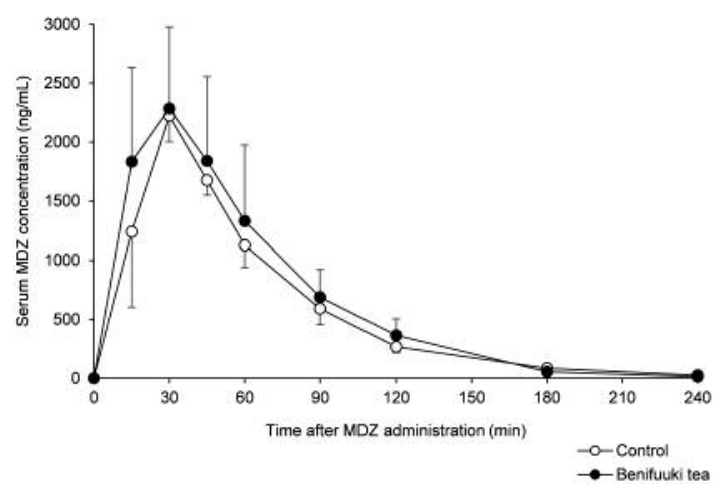

Figure 2. Serum concentration-time profiles of $M D Z$ after two treatments with Benifuuki tea. Benifuuki tea $(10 \mathrm{ml} / \mathrm{kg})$ or water (control) was orally administered to rats twice at 0.5 and $2 \mathrm{~h}$ before oral administration of $\mathrm{MDZ}(20 \mathrm{mg} / \mathrm{kg})$. Each point represents the mean \pm S.D. of 5 rats.

$(10,29)$. Therefore, it was hypothesized that an ingredient specific to Benifuuki tea, such as EGCG3"Me, was involved in the modulation of CYP2D activity. Accordingly, the inhibitory effects of Benifuuki tea and EGCG3"Me on CYP2D activity were then investigated in vitro. The results showed that Benifuuki tea strongly inhibited CYP2D activity in a concentration-dependent manner (Figure 4). However, the obtained $\mathrm{IC}_{50}$ value of $65 \mu \mathrm{M}$ for EGCG3"Me was equal to that for EGCG, suggesting that Benifuuki tea contains ingredients, other than these catechins, that inhibit CYP2D activity.

Mechanism-based inhibition (MBI) is an important process involved in drug interactions; in the present context, the inhibitory effect is considered to continue owing to the irreversible inactivation of CYP2D. The inhibition of CYP2D activity even 1 day after 1 week of tea treatment may be attributable to MBI of CYP2D by Benifuuki tea. Therefore, to clarify this issue, we conducted another in vitro study that included a preincubation step. The results showed no additional inhibition of BFL 1'-OH when Benifuuki tea was preincubated with rat liver microsomes and NADPH before the addition of BFL, indicating that MBI did not occur.

In this study, we were unable to clarify the mechanism by which Benifuuki tea inhibited CYP2D activity 1 day after 1 week of tea treatment. Although the inhibition observed in this experiment was not potent $(20 \%$ inhibition compared with that in the control group), an in vitro study revealed the potent direct inhibitory effect of Benifuuki tea on CYP2D activity. This finding suggests that a drug interaction occurs, depending on the timing of combination with drugs metabolized by CYP2D. Although CYP2D has a low abundance ratio in the liver, it actively participates in the metabolism of medicines, 
(A)

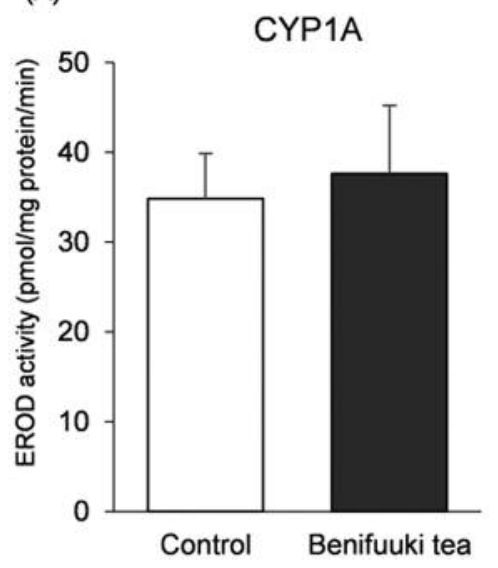

(D)

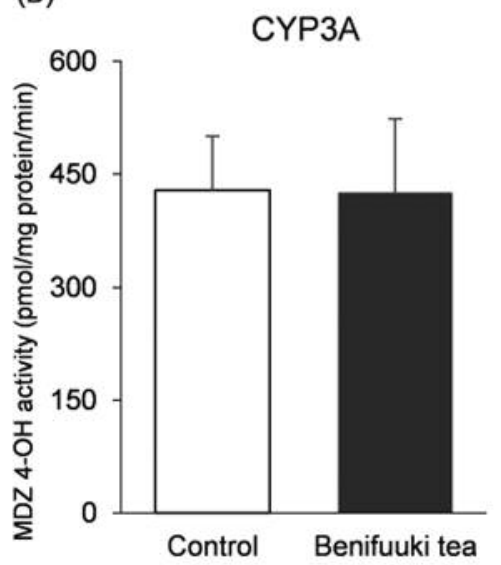

(G)

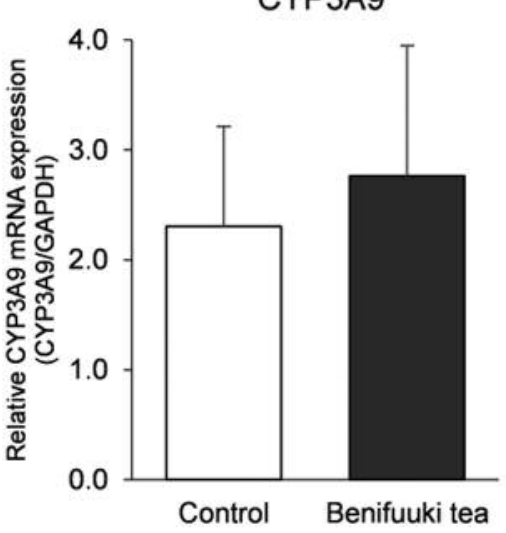

(B)

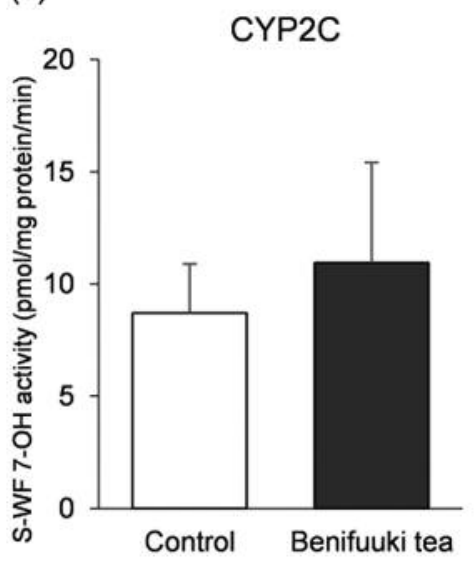

(E)

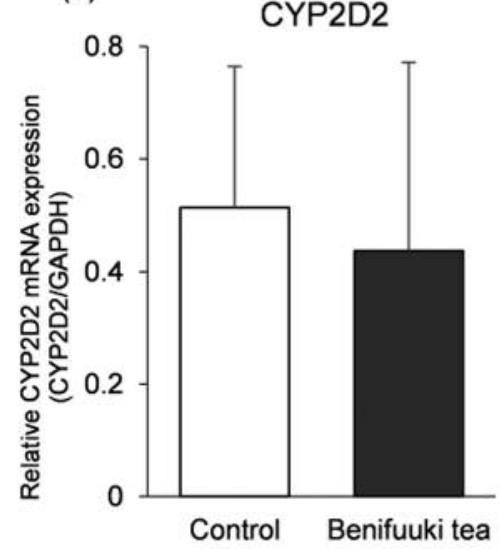

(C)

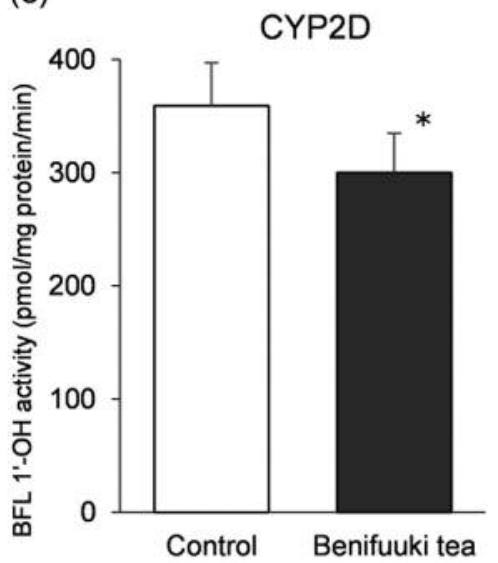

(F)

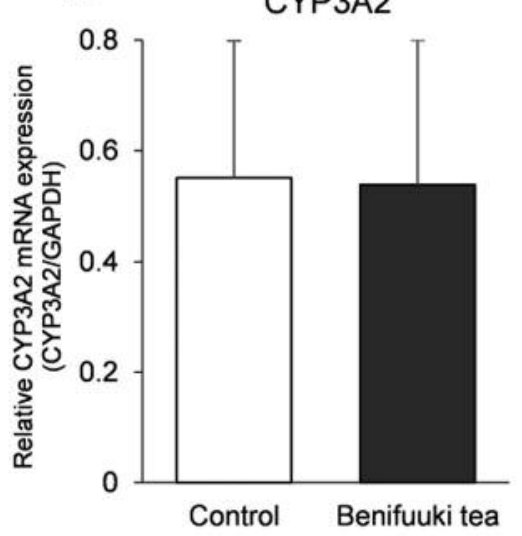

following that by CYP3A, therefore, it is important to investigate drug interactions mediated by CYP2D inhibition. Further in vivo studies are needed to clarify drug interactions with Benifuuki tea mediated by CYP2D inhibition, using a probe drug for this enzyme.
Figure 3. Effects of 1 week of oral administration of Benifuuki tea on CYP activities and mRNA expression. Liver and intestinal microsomes were prepared 1 day after the last treatment with $10 \mathrm{ml} / \mathrm{kg}$ Benifuuki tea or water (control) once a day for 1 week. The CYP activities were analyzed using the following reaction markers: CYP1A: ethoxyresorufin O-deethylation (EROD) (A), CYP2C: S-warfarin 7-hydroxylation ( $S$-WF 7-OH) (B), CYP2D: bufuralol 1'-hydroxylation (BFL 1'-OH) (C), and CYP3A: midazolam 4-hydroxylation (MDZ 4-OH) (D). Hepatic CYP2D2 (E), hepatic CYP3A2 (F), and intestinal CYP3A9 (G) mRNA levels were also determined. Data are shown as the mean $\pm S . D$. of values for 6 rats; *significant difference from the control $(p<0.05)$.

In the current study, we showed that Benifuuki tea was not likely to cause food-drug interactions mediated by CYP3A inhibition or induction when consuming a quantity obtained from normal drinking. However, weak inhibition of CYP2D activity was observed 1 day after 1 week of treatment with 
(A)

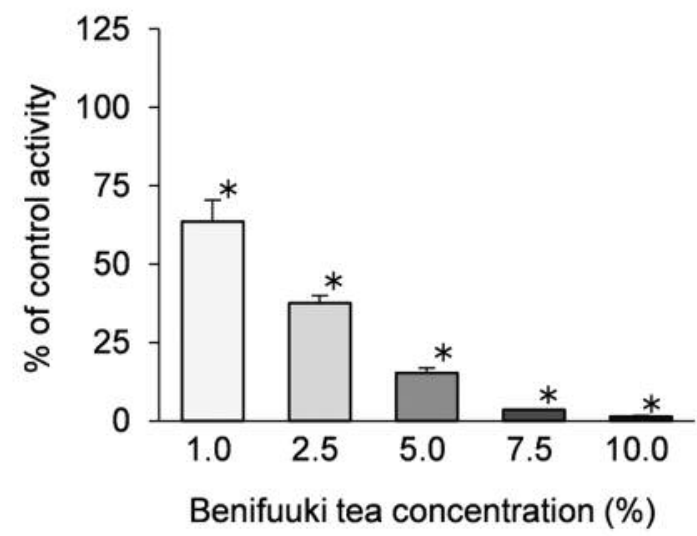

(C)

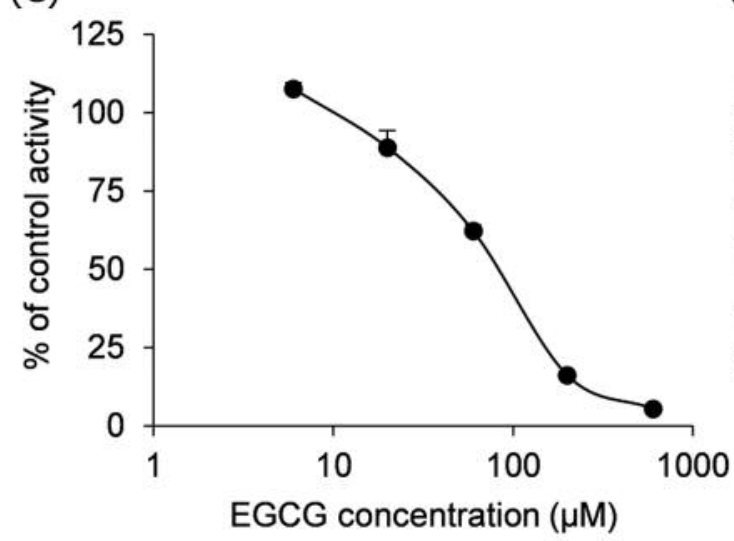

(B)

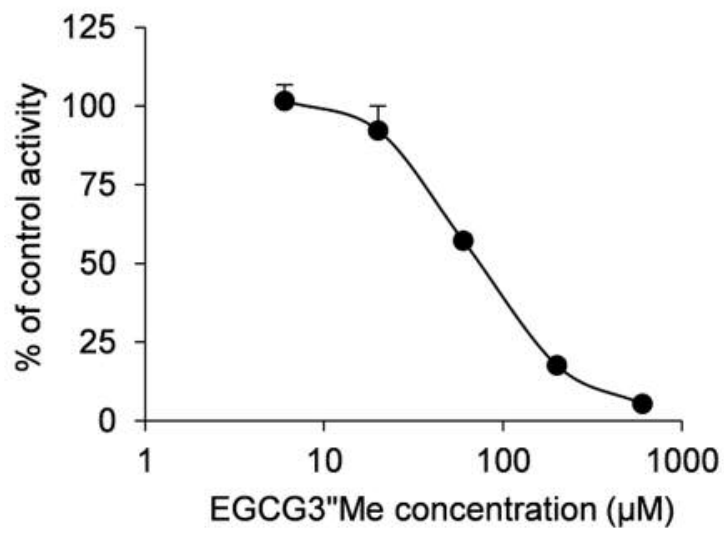

(D)

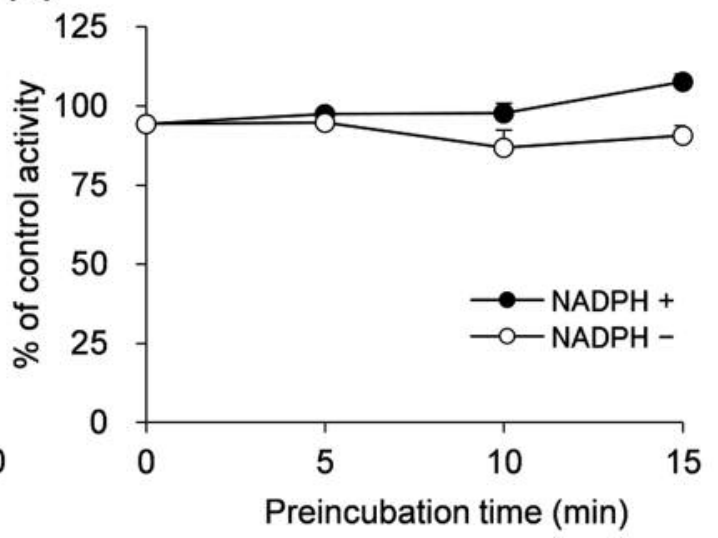

Figure 4. Inhibitory effects of Benifuuki tea, EGCG 3"Me, and EGCG on BFL 1'-OH activity in vitro. A direct inhibition study was performed with Benifuuki tea (A) (final concentration: 1-10\%), EGCG 3"Me (B), or EGCG (C) (final concentration: 6-600 $\mu M$ ) in pooled rat liver microsomes. The preincubation effect of Benifuuki tea $(0.5 \%)$ on the activity was studied using pooled rat liver microsomes in the presence (closed circles) or absence (opened circles) of NADPH (D). The mean \pm S.E. values of duplicate examinations are shown as a $\%$ of the control activity. $* p<0.05$ compared with control.

Benifuuki tea. In addition, in vitro hepatic CYP2D activity was strongly inhibited by the tea, suggesting the possibility of a food-drug interaction mediated by Benifuuki tea through the direct inhibition of CYP2D.

\section{Conflicts of Interest}

The Authors have no conflicts of interest to declare.

\section{References}

1 Okuda M: Epidemiology of Japanese cedar pollinosis throughout Japan. Ann Allergy Asthma Immunol 91: 288-296, 2003.

2 Ozasa K, Dejima K and Takenaka H: Prevalence of Japanese cedar pollinosis among schoolchildren in Japan. Int Arch Allergy Immunol 128: 165-167, 2002.

3 Tanaka A, Minoguchi K, Pawankar R and Adachi M: Asthma in patients with Japanese cedar pollinosis. World Allergy Organ J 5: 218-222, 2012 .
4 Maeda-Yamamoto M, Ema K, Tokuda Y, Monobe M, Tachibana $\mathrm{H}$, Sameshima Y and Kuriyama S: Effect of green tea powder (Camellia sinensis L. cv. Benifuuki) particle size on Omethylated EGCG absorption in rats; The Kakegawa Study. Cytotechnology 63: 171-179, 2011.

5 Maeda-Yamamoto $\mathrm{M}$, Inagaki $\mathrm{N}$, Kitaura $\mathrm{J}$, Chikumoto $\mathrm{T}$, Kawahara H, Kawakami Y, Sano M, Miyase T, Tachibana H, Nagai $\mathrm{H}$ and Kawakami T: O-methylated catechins from tea leaves inhibit multiple protein kinases in mast cells. J Immunol 172: 4486-4492, 2004.

6 Maeda-Yamamoto M, Ema K, Monobe M, Tokuda Y, Tachibana $\mathrm{H}$, Sameshima Y and Kuriyama S: Epicatechin-3-O-(3"-Omethyl)-gallate content in various tea cultivars (Camellia sinensis L.) and it's in vitro inhibitory effect on histamine release. J Agric Food Chem 60: 2165-2170, 2012.

7 Masuda S, Maeda-Yamamoto $M$, Usui $S$ and Fujisawa T: 'Benifuuki' green tea containing O-methylated catechin reduces symptoms of Japanese cedar pollinosis: a randomized, doubleblind, placebo-controlled trial. Allergol Int 63: 211-217, 2014. 
8 Bailey DG and Dresser GK: Interactions between grapefruit juice and cardiovascular drugs. Am J Cardiovasc Drugs 4: 281297, 2004.

9 Matsuda K, Nishimura Y, Kurata N, Iwase M and Yasuhara H: Effects of continuous ingestion of herbal teas on intestinal CYP3A in the rat. J Pharmacol Sci 103: 214-221, 2007.

10 Misaka S, Kawabe K, Onoue S, Werba JP, Giroli M, Tamaki S, Kan T, Kimura J, Watanabe $\mathrm{H}$ and Yamada S: Effects of green tea catechins on cytochrome P450 2B6, 2C8, 2C19, 2D6 and 3A activities in human liver and intestinal microsomes. Drug Metab Pharmacokinet 28: 244-249, 2013.

11 Nishikawa M, Ariyoshi N, Kotani A, Ishii I, Nakamura H, Nakasa $H$, Ida $M$, Nakamura $H$, Kimura $N$, Kimura $M$, Hasegawa A, Kusu F, Ohmori S, Nakazawa K and Kitada M: Effects of continuous ingestion of green tea or grape seed extracts on the pharmacokinetics of midazolam. Drug Metab Pharmacokinet 19: 280-289, 2004.

12 Muto S, Fujita K, Yamazaki Y and Kamataki T: Inhibition by green tea catechins of metabolic activation of procarcinogens by human cytochrome P450. Mutat Res 479: 197-206, 2001.

13 Oritani Y, Setoguchi Y, Ito R, Maruki-Uchida H, Ichiyanagi T and Ito T: Comparison of (-)-epigallocatechin-3-O-gallate (EGCG) and O-methyl EGCG bioavailability in rats. Biol Pharm Bull 36: 1577-1582, 2013

14 Maeda-Yamamoto M, Ema K and Shibuichi I: In vitro and in vivo anti-allergic effects of 'benifuuki' green tea containing $\mathrm{O}$ methylated catechin and ginger extract enhancement. Cytotechnology 55: 135-142, 2007.

15 Yang SP and Raner GM: Cytochrome P450 expression and activities in human tongue cells and their modulation by green tea extract. Toxicol Appl Pharmacol 202: 140-150, 2005.

16 Guengerich FP: Analysis and characterization of enzymes. In: Principles and Methods of Toxicology (Hayes AW (ed.). New York, Raven Press, pp. 1259-1313, 1994.

17 Lowry OH, Rosebrough NJ, Farr AL and Randall RJ: Protein measurement with the Folin phenol reagent. J Biol Chem 193: 265-275, 1951

18 Namba H, Nishimura Y, Kurata N, Iwase M, Hirai T and Kiuichi Y: Inhibitory effect of oxethazaine on midazolam metabolism in rats. Biol Pharm Bull 40: 1361-1365, 2017.

19 Saito Y, Nishimura Y, Kurata N, Iwase M, Aoki K and Yasuhara $\mathrm{H}$ : In vivo inhibition of CYP3A-mediated midazolam metabolism by anchusan in rats. J Pharmacol Sci 115: 399-407, 2011.
20 Tabata K, Yamaoka K, Kaibara A, Suzuki S, Terakawa M and Hata T: Moment analysis program available on Microsoft Excel $^{\circledR}$. Xenobio Metab Dispos 14: 286-293, 1999.

21 Yamaoka K, Tanigawara Y, Nakagawa T and Uno T: A pharmacokinetic analysis program (multi) for microcomputer. $\mathbf{J}$ Pharmacobiodyn 4: 879-885, 1981.

22 Williams FM, Mutch E, Woodhouse KW, Lambert D and Rawlins MD: Ethoxyresorufin O-deethylation by human liver microsomes. Br J Clin Pharmacol 22: 263-268, 1986.

23 Kronbach T, Mathys D, Gut J, Catin T and Meyer UA: Highperformance liquid chromatographic assays for bufuralol 1'hydroxylase, debrisoquine 4-hydroxylase, and dextromethorphan $\mathrm{O}$-demethylase in microsomes and purified cytochrome P-450 isozymes of human liver. Anal Biochem 162: 24-32, 1987.

24 Nishimura Y, Kurata N, Sakurai E and Yasuhara H: Inhibitory effect of antituberculosis drugs on human cytochrome P450mediated activities. J Pharmacol Sci 96: 293-300, 2004.

25 Wrighton SA and Ring BJ: Inhibition of human CYP3A catalyzed 1'-hydroxy midazolam formation by ketoconazole, nifedipine, erythromycin, cimetidine, and nizatidine. Pharm Res 11: 921-924, 1994

26 Feng WY: Metabolism of green tea catechins: an overview. Curr Drug Metab 7: 755-809, 2006.

27 Dürr D, Stieger B, Kullak-Ublick GA, Rentsch KM, Steinert HC, Meier PJ and Fattinger K: St. John's Wort induces intestinal P-glycoprotein/MDR1 an intestinal and hepatic CYP3A4. Clin Pharmacol Ther 68: 598-604, 2000.

28 Obach RS: Inhibition of human cytochrome P450 enzymes by constituents of St. John's wort, an herbal preparation used in the treatment of depression. J Pharmacol Exp Ther 294: 88-95, 2000.

29 Chow HH, Hakim IA, Vining DR, Crowell JA, Cordova CA, Chew WM, Xu MJ, Hsu CH, Ranger-Moore J and Alberts DS: Effects of repeated green tea catechin administration on human cytochrome P450 activity. Cancer Epidemiol Biomarkers Prev 15: 2473-2476, 2006. 